\title{
Empirical Aspects of Strand Displacement Amplification
}

\author{
G. Terrance Walker
}

Becton Dickinson Research Center, Research Triangle Park, North Carolina 27709-2016

s (SDA) is an isothermal, in vitro DNA amplification technique that is based on the ability of a restriction enzyme to nick the unmodified strand of a hemiphosphorothioate form of its recognition site and the ability of a DNA polymerase to initiate replication at the nick and displace the downstream nontemplate strand. Primers containing recognition sites for the nicking restriction enzyme bind to opposite strands of target DNA at positions flanking the sequence to be amplified. The target fragment is exponentially amplified by coupling sense and antisense reactions in which strands displaced from the sense reaction serve as a target for the antisense reaction and vice versa. A detailed mechanistic description of SDA using the restriction enzyme HinclI and an exonuclease-deficient form of the Klenow fragment of Escherichia coli DNA polymerase I (exoKlenow) is presented in Figure 1 and discussed elsewhere. ${ }^{(1,2)}$ Although the series of events depicted in Figure 1 may appear complicated, SDA operates by a very simple protocol: Target DNA is heatdenatured in the presence of all reagents except exo ${ }^{-}$Klenow and HincII. Amplification then proceeds at $\sim 37^{\circ} \mathrm{C}$ after cooling and addition of the enzymes.

Past publications on $\mathrm{SDA}^{(1,2)}$ presented data for a target sequence from Mycobacterium tuberculosis. Because DNA from $M$. tuberculosis is not readily available to most researchers, this review presents amplification of $\mathrm{pBR} 322$ so that the experiments can be repeated easily in any laboratory. The purpose of this review is to discuss empirical aspects of SDA using the $\mathrm{pBR} 322$ data as a guide for development of other SDA target systems.

\section{AMPLIFICATION OF PBR322}

Primer pairs were designed to amplify four regions of $\mathrm{pBR} 322$, ranging in length from 52 to $198 \mathrm{bp}$ (Fig. 1; Tables 1 and 2). We chose target sequences that are not present in popular cloning vectors (e.g., pUC19) to alleviate the problem of contaminating target DNA in the laboratory and enzyme preparations. $S_{1}$ was paired with either $S_{2 a}, S_{2 b}, S_{2 c}$ or $S_{2 d}$ so that the four target sequences would have one end in common. Single versions of $B_{1}$ and $B_{2}$ were used with all four target fragments by designing them to bind at target locations $5^{\prime}$ to those of $S_{1}$ and $S_{2 \mathrm{~d}}$. SDA reaction conditions were optimized for each target sequence with regard to primer sequences, organic solvents, and enzyme concentrations.

A series of amplification reactions was performed with varying amounts of target DNA from pBR322. In the case of all four target sequences, the level of amplified product is directly related to the initial amount of target (Fig. 2). This doseresponse is a result of competition between target-specific and background amplification. ${ }^{(1,2)}$ Regardless of initial target level, amplification proceeds until total amplified products (both targetspecific and background) reach a level at which further exponential amplification is attenuated because HincII is no longer in excess. ${ }^{(3)}$ Consequently, each sample in Figure 2 for a given target length exhibits a constant amplification factor independent of initial target concentrations. Background amplification increases with increasing amounts of nontarget DNA (human DNA in the present study), which means amplification factors decrease with increasing amounts of nontarget DNA. ${ }^{2}$
Samples containing no input target DNA exhibit target-specific bands in Figure 2 as a result of accidental contamination with $\sim 10$ amplicons (amplification product molecules from previous reactions), a common problem with sensitive amplification techniques. Targetspecific products were not detected in a sample that was heat-inactivated immediately after addition of HincII and exo ${ }^{-}$ Klenow.

Amplification factors decrease dramatically with increasing target length (Table 2). The strand displacement ability of exo- Klenow is expected to decrease with progressively longer targets. However, amplification factors decrease between 5- and 100-fold for each 50-bp increase in target length. Obviously, sequence, and not just length, is important. Within these target fragments, there may be local sequences that attenuate strand displacement replication. For example, Kong et al. ${ }^{(4)}$ reported that a DNA polymerase from Thermococcus litoralis halts replication at fairly specific sequence positions under strand displacement conditions. Alternatively, polymerases can switch from replicating the template strand to replicating the displaced strand, ${ }^{(5)}$ which produces a specific stop when the polymerase reaches the $5^{\prime}$ end of the displaced strand. We did not search for potential replication termination sites in these pBR322 sequences.

In addition, primer pairs can have short regions of homology that may allow one primer to be extended on the other, forming a primer-dimer. If this background priming occurs between $S_{1}$ and $\mathrm{S}_{2}$ at positions $3^{\prime}$ to the HincII site of each primer, a primer-dimer will be formed that is exponentially amplified 


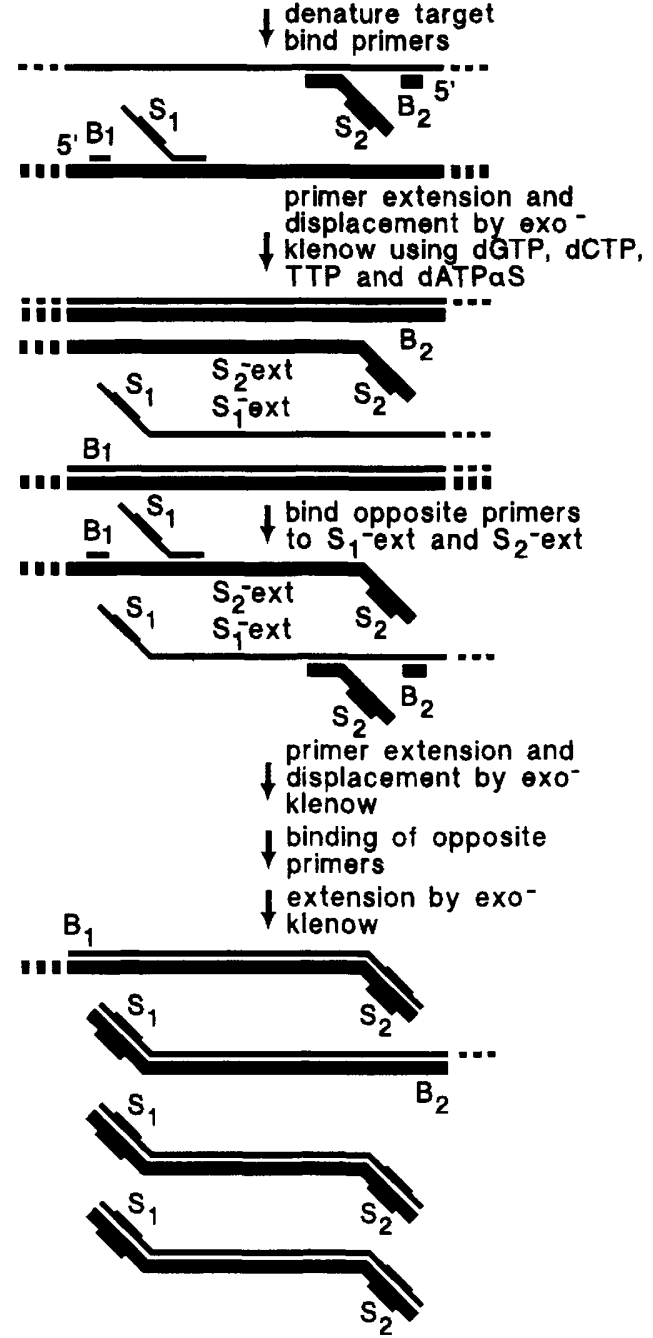

$\downarrow$ nicking by Hincll

extension/displacement $\checkmark$ by exo- klenow

$\downarrow$ binding of opposite primers

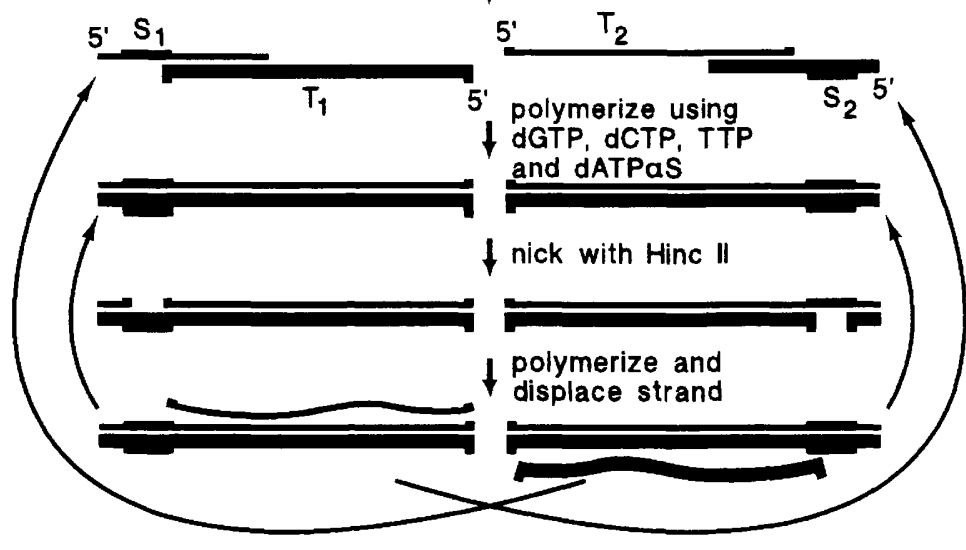

hybrldize SDA primers to displaced strands

because it contains a nickable HincII site at each end. These primer-dimers amplify very efficiently owing to their short length, thereby attenuating target-spe- cific amplification, especially for longer target sequences. Some of the $S_{1}$ and $S_{2 n}$ pairs in Table 2 may form primer-dimers more readily, which may also contribute
FIGURE 1 Schematic representation of SDA. The target DNA sample is heat-denatured in the presence of an excess of four primers $\left(B_{1}\right.$, $B_{2}, S_{1}$, and $\left.S_{2}\right) . S_{1}$ and $S_{2}$ contain target-binding regions at their $3^{\prime}$ ends. Each also has a recognition site ( $5^{\prime}$-GTTGAC) for the restriction enzyme HinclI located immediately $5^{\prime}$ to its target-binding region. $S_{1}$ and $S_{2}$ bind to opposite strands of the target sequence flanking the region to be amplified. $\mathrm{B}_{1}$ and $\mathrm{B}_{2}$ consist simply of target-binding sequences and bind at positions $5^{\prime}$ to those of $S_{1}$ and $S_{2}$. After the primers anneal at $37-40^{\circ} \mathrm{C}$, $\mathrm{HinclI}$ is added along with an exonuclease-deficient form of the Klenow fragment of $E$. coli DNA polymerase I (exo- Klenow). Exo ${ }^{-}$Klenow, which is present in excess over the number of target sequences, simultaneously extends all four primers using dGTP, dCTP, TTP, and dATP $\alpha$ S. A cascade of steps follows. $S_{1}$ and $S_{2}$ are extended and then displaced during extension of $B_{1}$ and $B_{2}$. The displaced extension products of $S_{1}$ and $S_{2}\left(S_{1}\right.$-ext and $S_{2}$-ext) serve as targets for the opposite primers. Further rounds of extension and displacement produce two fragments with a hemiphosphorothioate HincII site at each end and two longer fragments with a hemiphosphorothioate HincII site at just one end. HincII nicking and exo $^{-}$Klenow extension/displacement reactions initiate at these four fragments, automatically entering the reaction cycle at the bottom. During each cycle, the $3^{\prime}$ end of $\mathrm{S}_{1}$ binds to the $3^{\prime}$ end of the displaced target strand $T_{1}$, forming a duplex with $5^{\prime}$ overhangs. Likewise, $S_{2}$ binds to $\mathrm{T}_{2}$. $\mathrm{Exo}^{-}$Klenow extends the 3 ' ends of the duplexes producing hemiphosphorothioate recognition sites on $\mathrm{S}_{1} \cdot \mathrm{T}_{1}$ and $\mathrm{S}_{2} \cdot \mathrm{T}_{2}$. HincII nicks the unmodified primer strands of the hemiphosphorothioate recognition sites, leaving intact the modified complementary strands. Exo ${ }^{-}$Klenow extends the $3^{\prime}$ end at the nick on $S_{1} \cdot T_{1}$ and displaces the downstream strand that is equivalent to $T_{2}$. Likewise, extension at the nick on $S_{2} \cdot T_{2}$ results in displacement of $T_{1}$. Nicking and extension/displacement steps cycle continuously on $S_{1} \cdot T_{1}$ and $S_{2} \cdot T_{2}$ because extension at a nick regenerates a nickable HincII recognition site. Target amplification is exponential because strands displaced from $S_{1} \cdot T_{1}$ serve as target for $S_{2}$ whereas strands displaced from $S_{2} \cdot T_{2}$ serve as target for $S_{1}$. Sense and antisense strands are differentiated by thin and thick lines. Intact and nicked HincII recognition sequences are depicted by and 1 L. The partial HincII recognition sequence $5^{\prime}$-GAC and its complement 5 'GTC are present at the $5^{\prime}$ and $3^{\prime}$ ends of displaced strands as represented by $\mathbf{L}$ and $\boldsymbol{\Lambda}$.

to the lack of a smooth trend in the amplification factors as a function of target length.

Primer-dimers other than that be- 
TABLE 1 SDA Primer Sequences for Amplification of pBR322

\begin{tabular}{|c|c|c|}
\hline Primer & Sequence $^{a}$ & $\begin{array}{l}\text { pBR322 hybridization } \\
\text { (nucleotide positions) }\end{array}$ \\
\hline $\mathrm{S}_{1}$ & 5'-dTACAATAGTCCCAATCTTGTTGACGTTCCAGTAACC & $1801-1813$ \\
\hline $\mathrm{S}_{2 \mathrm{a}}$ & 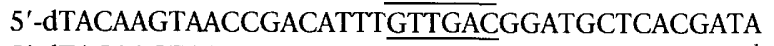 & $1852-1839$ \\
\hline $\mathrm{S}_{2 \mathrm{~b}}$ & $5^{\prime}$-dTACAAGTAACCGACTATTGTTGACGGAATTTCTGTTCA & $1896-1883$ \\
\hline $\mathrm{S}_{2 \mathrm{c}}$ & 5'-dTTGAAGTAACCGACTATT GTTGACGGCCATGTTAAGG & $1950-1938$ \\
\hline $\mathrm{S}_{2 \mathrm{~d}}$ & 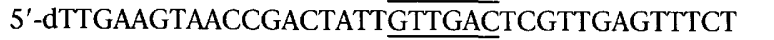 & $1998-1985$ \\
\hline $\mathrm{B}_{1}$ & 5'-dCCAGTTGTTTACCCT & $1781-1795$ \\
\hline $\mathrm{B}_{2}$ & 5'-dGCCTGTTCATCCG & $2018-2006$ \\
\hline
\end{tabular}

${ }^{a}$ Hincll recognition sites are underscored.

${ }^{b}$ See text for alternative sequence. tween $S_{1}$ and $S_{2}$ are not efficiently amplified. For example, a primer-dimer between $S_{1}$ and $B_{1}$ contains only a single HinclI site and, as such, is amplified in only a linear mode instead of exponentially. Furthermore, although a primerdimer between $S_{1}$ and $S_{1}$ (and likewise between $S_{2}$ and $S_{2}$ ) contains a nickable HincII site at each end, it is not expected to be exponentially amplified in an efficient manner because each displaced strand can form a hairpin conformation that hinders its capture by a new $S_{1}$ primer during each cycle of SDA (Fig. 1, bottom).

Inclusion of an organic solvent enhances amplification efficiency. Dimethyl sulfoxide (DMSO) is the optimal choice of organic solvent for all four pBR322 target sequences, although nearly equivalent results were obtained with slightly higher glycerol concentrations. Interestingly, the optimal DMSO concentration increases with increasing target length (Table 2). DMSO is expected to affect primer hybridization, strand displacement efficiency, and general enzyme activity. There is no clear trend in calculated melting temperatures $\left(T_{\mathrm{m}}\right)^{(6)}$ for $\mathrm{S}_{2 \mathrm{a}}-\mathrm{S}_{2 \mathrm{~d}}$, suggesting that opti- mal DMSO concentrations are not exclusively related to primer hybridization stability. The DMSO trend may reflect offsetting factors affecting strand displacement activity and general enzymatic activity; for example, exoKlenow may strand-displace more efficiently at higher DMSO concentrations, but the activity of HincII may decrease at higher DMSO concentrations. Consequently, amplification of the longer targets may benefit from enhanced strand displacement even though HincII activity is reduced.

The optimal exo ${ }^{-}$Klenow concentration for each of the target sequences increases with increasing target length (Table 2). Klenow is not highly processive, replicating only $\sim 8$ nucleotides per initiating event. ${ }^{(7)}$ When an exo- Klenow molecule dissociates from a template midstream during replication, another polymerase must bind quickly before the partially displaced downstream strand reanneals to the template. Therefore, it is not surprising that higher exo ${ }^{-}$ Klenow concentrations favor strand displacement replication of longer targets. An extremely simple model predicts that an infinitely high exo ${ }^{-}$Klenow concen-
TABLE 2 SDA Conditions for Amplification of pBR322

\begin{tabular}{lccllc}
\hline & $\begin{array}{l}\text { Percent } \\
\text { DMSO } \\
\text { (vol/vol) }^{\mathrm{b}}\end{array}$ & $\begin{array}{l}\text { exo } \\
\text { (units) }\end{array}$ & $\begin{array}{l}\text { Target } \\
\text { length } \\
\text { (base pairs) }^{\mathrm{c}}\end{array}$ & $\begin{array}{l}\text { Amplification } \\
\text { factor }\end{array}$ & $\begin{array}{l}\text { Duration } \\
\text { of SDA } \\
\text { (hr) }\end{array}$ \\
\hline $\mathrm{S}_{1}$ and $\mathrm{S}_{2 \mathrm{a}}$ & 6 & 3.5 & 52 & $4.5 \times 10^{8}$-fold & 2 \\
$\mathrm{~S}_{1}$ and $\mathrm{S}_{2 \mathrm{a}}$ & 6 & 3.5 & 52 & $8.1 \times 10^{8}$-fold & 3 \\
$\mathrm{~S}_{1}$ and $\mathrm{S}_{2 \mathrm{~b}}$ & 10 & 6 & 96 & $1.4 \times 10^{7}$-fold & 3 \\
$\mathrm{~S}_{1}$ and $\mathrm{S}_{2 \mathrm{c}}$ & 12 & 8 & 150 & $2.9 \times 10^{6}$-fold & 3 \\
$\mathrm{~S}_{1}$ and $\mathrm{S}_{2 \mathrm{~d}}$ & $\mathbf{1 5}$ & 10 & 198 & $3.0 \times 10^{4}$-fold & 3 \\
\hline
\end{tabular}

${ }^{a}$ All reactions also contained $\mathrm{B}_{1}$ and $\mathrm{B}_{2}$.

${ }^{b}$ Each reaction also contained $3 \%(\mathrm{vol} / \mathrm{vol})$ glycerol contributed by the enzyme stock solutions.

${ }^{c}$ Distance between target binding regions of $S_{1}$ and the indicated version of $S_{2}$. tration should be optimum regardless of target fragment length. However, exo ${ }^{-}$ Klenow concentrations higher than those indicated in Table 2 decrease amplification, probably because they promote mispriming and subsequent background amplification that competes with target amplification. Very high levels of exo- Klenow may also inhibit amplification by binding nonspecifically to target DNA.

All of the pBR322 amplification reactions were performed with 150 units of HincII. Higher levels of HincII were not pursued because of the inhibitory cost of the reagent. In the case of the 52-mer target, amplification factors decrease only $15 \%$ and $57 \%$ when the HincII level is reduced to 100 and 50 units, respectively for a 2-hr reaction using 3.5 units of exo ${ }^{-}$Klenow (data not shown). No attempt was made to reoptimize the exo- ${ }^{-}$Klenow concentration with lower HincII levels. Obviously, reasonable SDA results can be obtained with substantially lower HincII concentrations.

\section{GENERAL EMPIRICAL ASPECTS OF SDA}

\section{Primer and Target Sequences}

The sequences of $S_{1}$ and $S_{2}$ dramatically affect amplification efficiently. $S_{1}$ and $S_{2}$ usually have 11- to 14-nucleotide targetbinding regions with calculated $T_{\mathrm{m}}$ of $40-50^{\circ} \mathrm{C}$ for $0.5 \mu \mathrm{M}$ primer in $100 \mathrm{~mm}$ $\mathrm{NaCl}^{(6)}$ This length ensures stringency at $37-42^{\circ} \mathrm{C}$ in the presence of organic solvents. Exo ${ }^{-}$Klenow does not extend efficiently off primers with shorter target-binding regions. Longer targetbinding regions on $S_{1}$ and $S_{2}$ promote background amplification owing to mispriming on non-target DNA under the relatively low stringency conditions of SDA $\left(37^{\circ} \mathrm{C}\right)$. As mentioned previously, the $3^{\prime}$-ends of $S_{1}$ and $S_{2}$ must not bind, forming primer-dimers, because they are amplified efficiently at the cost of target amplification.

The $5^{\prime}$ sequences of $S_{1}$ and $S_{2}$ are not critical to performance. They simply must be long enough and thermodynamically stable enough to provide a priming site for exo ${ }^{-}$Klenow after nicking by HincII. However, it is important that the 5 ' sequences do not fortuitously promote background hybridization that competes with target-specific annealing. Historically, we either chose $5^{\prime}$ se- 


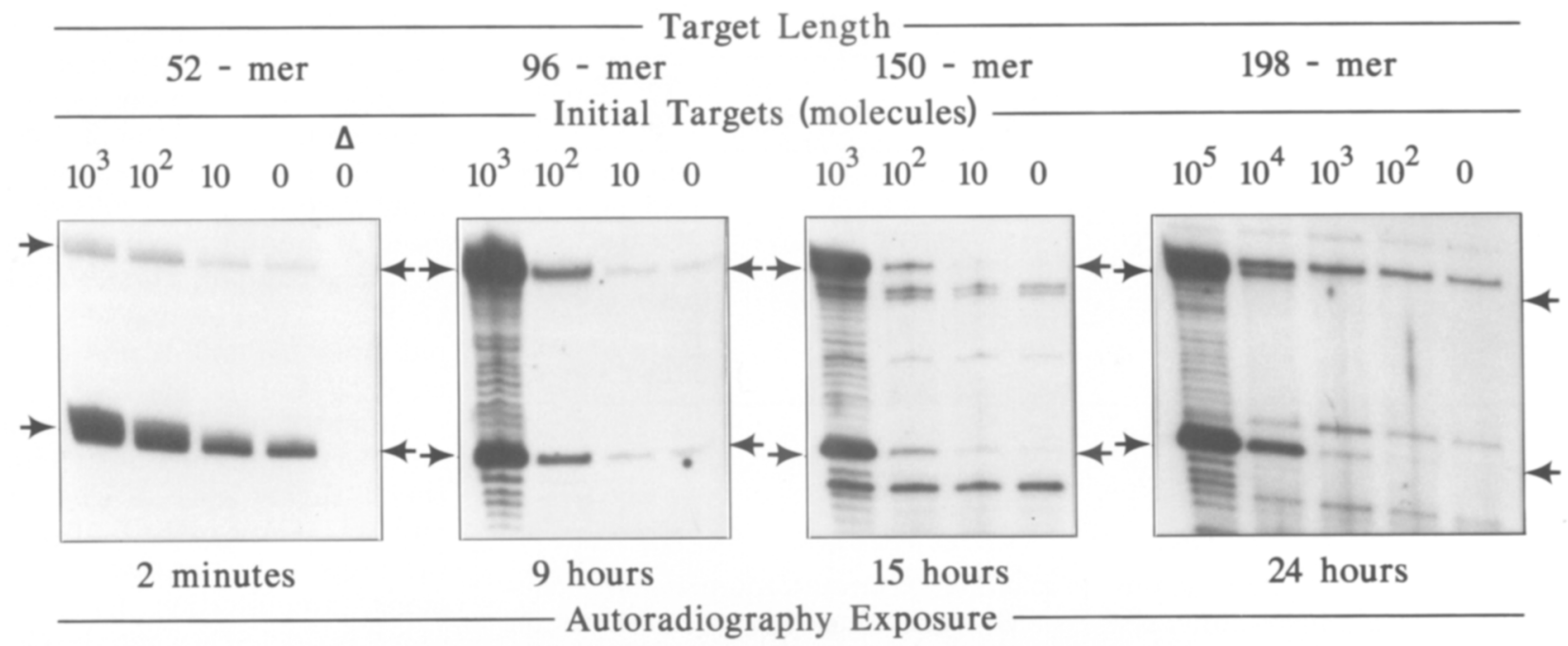

FIGURE 2 SDA of four pBR322 target sequences. The length of each pBR322 target fragment is indicated above each set of reactions. The initial number of pBR322 molecules present in each aliquot analyzed by gel electrophoresis is indicated above each lane. Reactions were performed generally as described previously ${ }^{(2)}$ and contained 150 units of HincIl (New England Biolabs), 1 mM each dGTP, dCTP, TTP, and dATP $\alpha$ S, 50 mM $\mathrm{K}_{\mathrm{i}} \mathrm{PO}_{4}(\mathrm{pH} 7.4), 6 \mathrm{mM} \mathrm{MgCl}_{2}, 3 \%$ (vol/vol) glycerol (from stock enzyme solutions), $0.1 \mathrm{mg} / \mathrm{ml}$ of BSA (acetylated, New England Biolabs), 50 ng of human placental DNA, 500 nM each $S_{1}$ and $S_{2}, 50$ nM each $B_{1}$ and $B_{2}$, and the indicated amounts of DMSO (Table 2), exo- Klenow (Table 2), and EcoRI-digested pBR322. (A 1- $\mu \mathrm{g} / 50 \mu \mathrm{l}$ stock solution of pBR322 was linearized with EcoRI to facilitate primer binding.) Samples were heat-denatured for $2 \mathrm{~min}$ at $95^{\circ} \mathrm{C}$, and $\mathrm{HinclI}$ and exo ${ }^{-}$Klenow were added after the samples were incubated for $\sim 2 \mathrm{~min}$ at $37^{\circ} \mathrm{C}$. After amplification for $3 \mathrm{hr}$ at $37^{\circ} \mathrm{C}$, samples were heat-inactivated for $2 \mathrm{~min}$ at $95^{\circ} \mathrm{C} .(\triangle) \mathrm{A}$ "no-amplification" sample that was treated for 2 min at $95^{\circ} \mathrm{C}$ immediately after addition of exo- Klenow and HincII. An aliquot $(5 \mu \mathrm{l})$ from each reaction was mixed with $7 \mu \mathrm{l}$ of $50 \mathrm{mM} \mathrm{K}_{\mathrm{i}} \mathrm{PO}_{4}(\mathrm{pH} 7.4) ; 1 \mathrm{~mm}$ each dGTP, dCTP, TTP, and $\mathrm{dATP} \alpha$; $0.1 \mathrm{mg} / \mathrm{ml}$ of BSA; $6 \mathrm{mM} \mathrm{MgCl}{ }_{2} ; 0.3 \mu \mathrm{M} 5^{\prime}{ }^{32} \mathrm{P}$-probe (5'-dGTTACTGATGATGAAC); $50 \mathrm{~mm}$ Tris (pH 8); and $10 \mathrm{~mm} \mathrm{MgCl}_{2}$. [The ${ }^{32} \mathrm{P}$-probe (1 $\mu \mathrm{M}$ ) was labeled with $50 \mu \mathrm{l}$ of $50 \mathrm{mM}$ Tris ( $\mathrm{pH} 8$ ), $10 \mathrm{mM} \mathrm{MgCl}_{2}, 50$ units of T4 polynucleotide kinase (New England Biolabs), and $350 \mu \mathrm{Ci}$ of $\left[\gamma_{-}{ }^{32} \mathrm{P}\right]$ ATP (New England Nuclear) and was used without subsequent purification.] The ${ }^{32} \mathrm{P}$-probe anneals to amplified strands containing the $S_{1}$ sequence at their $5^{\prime}$ ends (pBR322 nucleotide positions 1835-1820). Samples were then heated for 2 min at $95^{\circ} \mathrm{C}$ followed by 2 min at $37^{\circ} \mathrm{C}$. An aliquot of $2 \mu \mathrm{l}$ of $0.5 \mathrm{U} / \mu \mathrm{l}$ of Klenow (Boehringer Mannheim, sequencing grade) was added, and the samples were incubated for 10 min at $37^{\circ} \mathrm{C}$. Samples were mixed with $14 \mu \mathrm{l}$ of $50 \%$ (wt/vol) urea, $20 \mathrm{mM} \mathrm{Na}{ }_{2}$ EDTA, and $0.05 \%$ bromphenol blue/xylene cylanol, and were analyzed by $8 \%$ denaturing gel electrophoresis (Acryl-a-Mix 8 from Promega). X-ray film (Fuji RX Film) was exposed for the indicated times using intensifying screens. Exposures $>2$ min were performed at $-70^{\circ} \mathrm{C}$. Amplification factors (Table 2) were estimated as described previously. ${ }^{(2)}$ Regardless of which version of $\mathrm{S}_{2 n}$ is paired with $\mathrm{S}_{1}$, two ${ }^{32} \mathrm{P}$-labeled extension products are produced, (indicated by arrows). A 38-mer product (lower band) results from extension of the ${ }^{32} \mathrm{P}$-labeled detector probe on a strand containing a nicked HincII site, whereas the upper 58-mer band corresponds to a target strand with an unnicked HincII site (target strand with a full-length $S_{1}$ sequence at its $5^{\prime}$ end).

quences that did not form obvious interor intraprimer secondary structures or we designed $S_{1}$ and $S_{2}$ to anneal specifically to one another at their 5 '-ends. ${ }^{(1,2)}$ 5 ' Homology was designed to block potentially damaging secondary structures, although it does not necessarily protect $S_{1}$ and $S_{2}$ from pernicious hairpin or duplex formation.

The sequences of $B_{1}$ and $B_{2}$ are not critical to amplification efficiency. However, they must not anneal to any of the other four primers $\left(B_{1}, B_{2}, S_{1}\right.$, or $\left.S_{2}\right)$ in a manner that attenuates target-specific hybridization.

The target sequence must not naturally contain recognition sites for the nicking restriction enzyme used in SDA. Fortunately, HincII appears to retain specificity for its exact recognition sequence, even under SDA conditions containing organic solvents that are known to promote cleavage at sequences closely resembling recognition sites ("star" sites). (These four pBR322 target sequences contain a number of potential star HincII sites.) Therefore, only exact HincII recognition sites in the target sequence are of concern. However, it is important to search for all four HincIl sites (5'-GTPyPuAC).

\section{Primer Concentrations}

$S_{1}$ and $S_{2}$ concentrations of $500 \mathrm{~nm}$ ensure target binding on a second time scale. Lower concentrations render target hybridization rate limiting during each SDA cycle (Fig. 1, bottom). Higher concentrations needlessly increase the potential for background amplification. $B_{1}$ and $B_{2}$ are typically used at lower concentrations $(50 \mathrm{nM})$ to ensure that they do not anneal and extend before $S_{1}$ and $\mathrm{S}_{2}$. During the entire SDA incubation pe- riod, $B_{1}$ and $B_{2}$ extend only twice at the beginning.

\section{Organic Solvents}

Organic solvents generally destabilize nucleic acid hybridization. Inclusion of organic solvents enhances amplification, probably because it raises the stringency of primer hybridization and facilitates strand displacement. Favorable results are usually obtained with $2-5 \%$ 1-methyl 2-pyrrolidinone, 5-20\% glycerol or $5-20 \%$ DMSO. Adequate results can generally be obtained with either of the three organic solvents over a broad concentration range. However, the optimal choice of organic solvent is target dependent.

\section{Enzyme Sources and Concentrations}

Exo $^{-}$Klenow is commercially available only from U.S. Biochemical at stock con- 
centrations of 5-10 U/ $\mu \mathrm{l}$. We purchase HincII at high concentration (50-75 $\mathrm{U} / \mu \mathrm{l})$ from New England Biolabs and Life Technologies, Inc. HindII, which is identical to HincII except from a different strain of Haemophilus influenzae, is available from Boehringer Mannheim at $>40$ $\mathrm{U} / \mu \mathrm{l}$. The unit activity of these enzymes may vary between lots, so a quick concentration optimization experiment should be performed with each new lot.

The ratio of exo ${ }^{-}$Klenow to HincII is important in addition to the absolute concentration of each. After HincII dissociates from a nicked site, exo- Klenow should extend the nick; HincII binding to the nicked site would be unproductive. Six units of exo ${ }^{-}$Klenow and 150 units of HincII (the average concentrations used in this study) correspond to $\sim 10^{12}$ and $10^{13}$ molecules. An extremely simple mechanistic model predicts that a $1: 10$ ratio of exo ${ }^{-}$Klenow to HincII would not ensure that $\mathrm{exo}^{-}$Klenow binds first to the nicked site following HincII dissociation. Because exo- Klenow is not expected to have a particular affinity for an unnicked HincII recognition sequence, one might expect that high exo- Klenow/Hincll ratios would be optimal were it not for increased background amplification at high exo ${ }^{-}$ Klenow levels. The exo ${ }^{-}$Klenow concentration must also not be so high that it nonspecifically blocks HincII access to its recognition site. Obviously, complicated factors must be considered to explain optimal polymerase and restriction enzyme concentrations.

Exo $^{-}$Klenow tends to add extra nucleotides to the end of duplex DNA (terminal transferase activity). ${ }^{(8)}$ Addition of extra nucleotides is a particular concern in SDA because displaced strands must be subsequently extended on the primer $\left(S_{1}\right.$ or $\left.S_{2}\right)$ that captures it. If a displaced strand contains an extra $3^{\prime}$ nucleotide, it will base-pair with the underlined $\mathrm{T}(\underline{\mathrm{T}})$ in the HincII site $5^{\prime}$-GTTGAC on $S_{1}$ or $S_{2}$. In the presence of dGTP, dCTP, TTP, and $\mathrm{dATP} \alpha \mathrm{S}$, exo ${ }^{-}$Klenow adds $\mathrm{dA} \alpha \mathrm{S}$ and $\mathrm{dG}$ with approximately equal tendency to the ends of duplex DNA. ${ }^{(3)}$ An extra 3'$\mathrm{dA} \alpha \mathrm{S}$ on the displaced strand will naturally base-pair with the $\underline{T}$ in the HinclI site 5'-GTTGAC. In contrast, however, an extra $3^{\prime}-\mathrm{dG}$ on the displaced strand will form a dG. $T$ base pair in the HincII site. Such a dG ' T mismatch at the $3^{\prime}$ end of the displaced strand may impede extension on the SDA primer. Furthermore, the resultant $\mathrm{dG} \cdot \underline{\mathrm{T}}$-containing HincII site may not be efficiently nicked (it may undergo double-stranded cleavage ${ }^{9}$ ). Therefore, addition of an extra dG to the 3 ' end of the duplex may produce a displaced strand that does not serve as target, thereby nullifying that cycle of SDA (Fig. 1, bottom).

However, buffer conditions can be adjusted such that extra nucleotides are not efficiently added to the 3 ' ends of duplexes. Therefore, this extra nucleotide phenomenon is not necessarily detrimental to SDA, although it should be considered when designing SDA systems that use a restriction enzyme other than HincII. For example, NciI can be used in conjunction with the recognition site $5^{\prime}$-CCGGG using dATP, TTP, dGTP, and dCTP $\alpha$ S. $^{(1)}$ Addition of an extra $3^{\prime}$-dA to the ends of displaced strands, which is highly favored over an extra $3^{\prime}$ $\mathrm{dG}$, would produce a $\mathrm{dC} \cdot \mathrm{dA}$ mismatch at the underlined $\mathrm{C}(\underline{\mathrm{C}})$ in the NciI site $5^{\prime}$-CCGGG.

Klenow, which possesses $3^{\prime}$ to $5^{\prime}$ exonuclease activity, does not add extra nucleotides to the ends of duplexes. ${ }^{(8)}$ However, Klenow does not work as well as exo ${ }^{-}$Klenow in SDA because its $3^{\prime}$ to $5^{\prime}$ exonuclease activity degrades free primers and promotes mispriming on nontarget DNA.

\section{Buffer/Salt Conditions}

SDA reactions usually contain $50 \mathrm{~mm}$ $\mathrm{K}_{\mathrm{i}} \mathrm{PO}_{4}(\mathrm{pH} 7.4)$ which serves as a buffer and $\mathrm{K}^{+}$source for the enzymes. In preparing this buffer, one must be careful not to raise the $\mathrm{K}^{+}$concentration any higher than necessary while adjusting the $\mathrm{pH}$ because $\mathrm{K}^{+}$concentrations $>100$ $\mathrm{mm}$ are detrimental to SDA. We mix 13 $\mathrm{ml}$ of $0.5 \mathrm{M} \mathrm{KH}_{2} \mathrm{PO}_{4}$ with $87 \mathrm{ml}$ of $0.5 \mathrm{M}$ $\mathrm{K}_{2} \mathrm{HPO}_{4}$ to form $0.5 \mathrm{M} \mathrm{K}_{\mathrm{i}} \mathrm{PO}_{4}(\mathrm{pH} 7.6)$ (10× stock solution), which corresponds to a final $1 \times$ concentration of $94 \mathrm{mM} \mathrm{K}^{+}$ in the SDA reaction. The $\mathrm{pH}$ of this $10 \times$ stock solution decreases to $\mathrm{pH} \sim 7.4$ upon dilution to the final $1 \times$ concentration of $50 \mathrm{~mm} \mathrm{~K}_{\mathrm{i}} \mathrm{PO}_{4}$. Changes in $\mathrm{pH}$ from 7.4 to 7.6 have minimal effect.

An earlier version of SDA used $50 \mathrm{~mm}$ Tris- $\mathrm{HCl}(\mathrm{pH} 7.4), 50 \mathrm{~mm} \mathrm{NaCl}$, and 50 $\mathrm{mm} \mathrm{KCl}$ instead of $50 \mathrm{mM} \mathrm{K}_{\mathrm{i}} \mathrm{PO}_{4}(\mathrm{pH}$ 7.4). ${ }^{(1)}$ Use of $\mathrm{K}_{\mathrm{i}} \mathrm{PO}_{4}$ produces higher amplification factors. However, a drawback in using $\mathrm{K}_{\mathrm{i}} \mathrm{PO}_{4}$ is that magnesium precipitates with phosphate at concentrations higher than the final SDA concentrations. Consequently, when assembling an SDA reaction sample, $\mathrm{MgCl}_{2}$ is not added until all other reagents except enzymes have been added to ensure that $\mathrm{K}_{\mathrm{i}} \mathrm{PO}_{4}$ and $\mathrm{MgCl}_{2}$ do not encounter one another at elevated concentrations.

SDA reactions usually contain $6 \mathrm{~mm}$ $\mathrm{MgCl}_{2}$ and $4 \mathrm{~mm}$ total dNTP. The free $\mathrm{Mg}^{2+}$ concentration is $\sim 2 \mathrm{mM}$ because dNTPs quantitatively bind $\mathrm{Mg}^{2+}$. Other concentration combinations of $\mathrm{MgCl}_{2}$ and dNTPs may produce favorable results. However, because the dNTP and free $\mathrm{Mg}^{2+}$ concentrations are coupled, the two concentrations cannot be changed independently. The reader should also be aware that precipitation of magnesium phosphate may be more prevalent at other concentrations of $\mathrm{MgCl}_{2}$, dNTPs, and $\mathrm{K}_{\mathrm{i}} \mathrm{PO}_{4}$.

Bovine serum albumin (BSA) is usually included at a final concentration of $0.1 \mathrm{mg} / \mathrm{ml}$ to stabilize enzymes and prevent their adsorption to polypropylene microcentrifuge tubes, although indication of its inclusion was inadvertently omitted from previous publications. ${ }^{(1,2)}$ Amplification of the 52-mer pBR322 target decreases $23 \%$ for a 2 -hr reaction when addition of BSA is omitted. However, the final BSA concentration for this reaction was still $0.6 \mu \mathrm{g} / 50 \mu \mathrm{l}$ as a result of a contribution from the stock vendor solution of HincII.

\section{Temperature}

Reactions are usually performed at $37-$ $42^{\circ} \mathrm{C}$. Higher temperatures reduce the stability of HincII and exo ${ }^{-}$Klenow. Lower temperatures enhance background amplification due to mispriming. The SDA sample must be at its incubation temperature $\left(37-42^{\circ} \mathrm{C}\right)$ before addition of $\mathrm{HincII}$ and exo- Klenow. Assembly of reactions at lower temperatures promotes mispriming and, thus, background amplification, which attenuates target-specific amplification.

The optimal organic solvent concentration usually decreases with higher temperature, as both destabilize nucleic acid hybridization. Often, a few combinations of temperature and organic solvent produce equivalent results. Temperatures higher than $37^{\circ} \mathrm{C}$ were not tried with these pBR322 targets.

The target denaturation step at $\sim 95^{\circ} \mathrm{C}$ before addition of $\mathrm{HinCII}$ and exo $^{-} \mathrm{Kle}-$ now should be limited to $\sim 2 \min$ to 
guard against magnesium phosphate precipitation, which is promoted at high temperature. However, it is essential that the sample actually achieves $\sim 95^{\circ} \mathrm{C}$ during that 2-min incubation to denature the target fully.

\section{Time}

SDA typically converts from an exponential to a linear mode after $\sim 2 \mathrm{hr}$ because at that point amplified product (both target-specific and background) reaches a level that renders HincII no longer in excess. ${ }^{(1,3)}$ The reactions in Figure 2 were performed for $3 \mathrm{hr}$ to reach the highest level of amplified product with the longer targets that do not amplify as well. However, the extra hour produced only about twofold more product (Table 2).

Each SDA cycle (Fig. 1, bottom) coñsists of (1) primer binding to a displaced target fragment, (2) extension of the primer/target complex by exo- Klenow, (3) nicking of the resultant hemiphosphorothioate HinclI site, (4) dissociation of HinclI from the nicked site, and (5) extension of the nick and displacement of the downstream strand by exo ${ }^{-}$Klenow. For short target fragments $(\sim 50 \mathrm{nu}-$ cleotides), the cycle time is $\sim 3.5 \mathrm{~min}$, with the rate-limiting step being HincII dissociation from the nicked site (G.T. Walker, unpubl.). The extension/displacement step may become somewhat rate limiting for longer target fragments ( $>100$ nucleotides). However, rate-limiting extension/displacement behavior would probably reflect inefficient displacement of longer fragements owing to poor processivity of $\mathrm{exo}^{-}$Klenow rather than a slow intrinsic rate of nucleotide incorporation. Low processivity during extension/displacement requires multiple polymerase initiation events. Each initiation event must compete with reannealing of the displaced strand reannealing to the template strand.

\section{SUMMARY}

The most attractive feature of SDA is its operation at a single temperature, which removes the need for instrumented temperature cycling as with PCR and the ligase chain reaction. ${ }^{(10)}$ Highly reproducible temperature profiles, over a large array of samples, can burden the accuracy and expense of an amplification technique. However, the expense of a temperature cycler is offset somewhat by the cost of additional enzymes used in isothermal techniques. In comparisons with isothermal, transcription-based techniques, ${ }^{(11)}$ SDA requires fewer enzymes and has a simpler mechanism. SDA may also be more robust than transcription-based processes because it is not susceptible to contaminating ribonuclease activity. This is generally more of a concern when using clinical samples.

The most significant disadvantage of SDA is its inability to efficiently amplify long target sequences. Until this shortcoming is eliminated, SDA will be assigned to the diagnostic laboratory along with the ligase chain reaction. Currently, SDA cannot compete with PCR in research applications such as the isolation of gene sequences. The second disadvantage of SDA is that it operates at relatively low (nonstringent) temperatures, which produces considerable background reactions. Consequently, SDA reaction products cannot be analyzed routinely by ethidium-stained gel electrophoresis, as is used commonly with PCR, unless the target sample contains a large number of initial targets.

\section{NOTE}

Just before submission of this article, I was informed that the updated sequence of pBR322 $2^{(12)}$ varies from the published sequence on which I designed the SDA primers. ${ }^{(13)}$ The updated sequence contains a C instead of a $\mathrm{T}$ at position 1894 and single-nucleotide deletions at positions 1899 and 1915 (nucleotide positions as in ref. 13). Unfortunately, primer $S_{2 b}$ binds at one of the discrepant regions. According to the updated sequence, ${ }^{(12)}$ primer $S_{2 b}$ should be 5'-dTACAAGTAACCGACTATTGTTFACGGGATTTCTGTTCA. Previous experience suggests that a single mismatch toward the 5 '-end of the target-binding region of $\mathrm{S}_{2 b}$ should reduce SDA efficiency by 2 - to 10 -fold. Therefore, the amplification factor for the $S_{1} / S_{2 b}$ primer pair in Table 2 may improve with the above updated version of $S_{2 b}$. The binding positions of primers $S_{2 c}, S_{2 d}$, and $B_{2}$ in Table 1 reflect the updated pBR322 sequence. $^{(12)}$

\section{REFERENCES}

1. Walker, G.T., M.C. Little, J.G. Nadeau, and D.D. Shank. 1992. Isothermal in vitro amplification of DNA by a restriction enzyme/DNA polymerase system. Proc. Natl. Acad. Sci. 89: 392-396.

2. Walker, G.T., M.S. Fraiser, J.L. Schram, M.C. Little, J.G. Nadeau, and D.P. Malinowski. 1992. Strand displacement amplification-An isothermal, in vitro DNA amplification technique. Nucleic Acids Res. 20: 1691-1696.

3. J. Nadeau, personal communication.

4. Kong, H., R.B. Kucera, and W.E. Jack. 1993. Characterization of a DNA polymerase from the hyperthermophile archaea Thermococcus litoralis. J. Biol. Chem. 268: 1965-1975.

5. Nossal, N.G. and B.M. Peterlin. 1979. DNA replication by bacteriophage T4 proteins. J. Biol. Chem. 254: 6032-6037.

6. The computer program OLIGO by $\mathrm{Na}$ tional Biosciences, Hamel, MN.

7. Polesky, A.H., T.A. Steitz, N.D. Grindley, and C.M. Joyce. 1990. Identification of residues critical for the polymerase activity of the klenow fragment of DNA polymerase I from Escherichia coli. J. Biol. Chem. 265: 14579- 14591.

8. Clark, J.M., C.M. Joyce, and G.T. Beardsley. 1987. Novel blunt-end addition reactions catalyzed by DNA polymerase I of Escherichia coli. J. Mol. Biol. 198: 123-127.

9. Jiricny, J. and D. Martin. 1986. Restriction endonucleases HindII and TaqI cleave DNA with mismatched nucleotides within their recognition sites. Nucleic Acids Res. 14: 1943-1949.

10. Barany, F. 1991. The ligase chain reaction in a PCR world. PCR Methods Applic. 1: 516.

11. Fahy, E., D.Y. Kwoh, and T.R. Gingeras. 1991. Self-sustained sequence replication (3SR): An isothermal transcription-based amplification system alternative to PCR. PCR Methods Applic. 1: 25-33.

12. N. Watson. 1988. A new revision of the sequence of plasmid pBR322. Gene 70: 399-403; NCBI-GenBank Flat File Release 74.0, National Center for Biotechnology Information, National Library of Medicine 38A 8N805, 8600 Rockville Pike, Bethesda, MD 20894.

13. Maniatis, T., E.F. Fritsch, and J. Sambrook. 1982. Molecular cloning: A laboratory manual. Cold Spring Harbor Laboratory, Cold Spring Harbor, New York. 


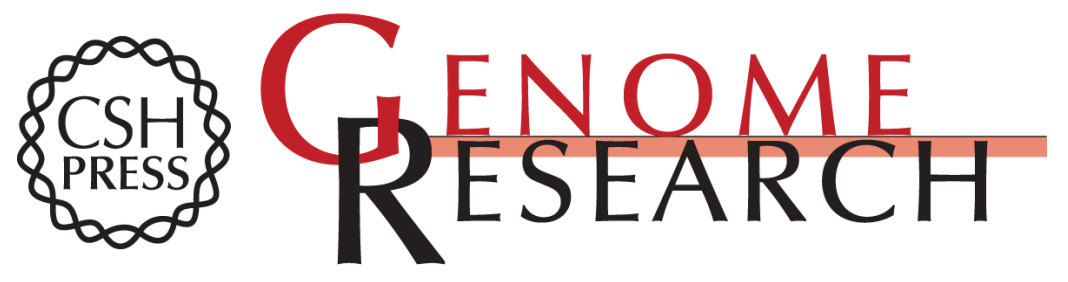

\title{
Empirical aspects of strand displacement amplification.
}

\author{
G T Walker
}

Genome Res. 1993 3: 1-6

References This article cites 10 articles, 4 of which can be accessed free at: http://genome.cshlp.org/content/3/1/1.full.html\#ref-list-1

\section{License}

Email Alerting Receive free email alerts when new articles cite this article - sign up in the box at the Service top right corner of the article or click here.

\section{Affordable, Accurate Sequencing.}

To subscribe to Genome Research go to: https://genome.cshlp.org/subscriptions 